\title{
Archéopages
}

Archéopages

Archéologie et société

37 | 04/2013

Jardins

\section{Regards aériens sur quelques parcs et jardins de châteaux modernes. Aménagements paysagers des $\mathrm{XVI}^{\mathrm{e}}, \mathrm{XVII}^{\mathrm{e}}$ et $\mathrm{XVIII}^{\mathrm{e}}$ siècles dans l'ouest de la France}

\section{Gilles Leroux}

\section{CpenEdition}

\section{Journals}

Édition électronique

URL : https://journals.openedition.org/archeopages/350

DOI : 10.4000/archeopages.350

ISSN : 2269-9872

Éditeur

INRAP - Institut national de recherches archéologiques préventives

Édition imprimée

Date de publication : 1 février 2014

Pagination : 52-61

ISSN : $1622-8545$

\section{Référence électronique}

Gilles Leroux, «Regards aériens sur quelques parcs et jardins de châteaux modernes. Aménagements paysagers des $\mathrm{xvI}^{\mathrm{e}}, \mathrm{xvII}^{\mathrm{e}}$ et xvIII siècles dans l'ouest de la France », Archéopages [En ligne], 37 | 04/2013, mis en ligne le 01 avril 2015, consulté le 22 janvier 2022. URL : http://journals.openedition.org/ archeopages/350; DOI : https://doi.org/10.4000/archeopages.350 


\section{Regards aériens sur quelques parcs et jardins de châteaux modernes Aménagements paysagers des $X V I^{e}, x V I^{e}$ et $x V I I^{e}$ siècles dans l'ouest de la France}

Gilles LerouX Inrap, UMR 6566, CReAAH

À l'occasion du quatrième centenaire de la naissance d'André Le Nôtre, nous avons recherché dans nos archives photographiques des exemples d'aménagements paysagers associés à des châteaux modernes, c'est-à-dire contemporains à peu de choses près du Grand Siècle. À défaut de plans terriers de l'époque moderne, les premiers plans cadastraux du début du XIX ${ }^{\mathrm{e}}$ siècle relatifs à ces sites ont ensuite été recherchés en vue d'interpréter les photographies aériennes.

L'archéologie aérienne, cantonnée à la marge des disciplines historiques ou archéologiques, n'a pas prêté jusqu'à présent une attention suffisante à l'identification de ce type de vestiges. En outre, sa pratique requiert des conditions météorologiques spécifiques : l'observation des vestiges, très légers pour ceux qui nous concernent ici, dépend d'une conservation de leurs fondations dans le sous-sol ; leur lisibilité est facilement remise en cause par la nature des éventuelles cultures couvrantes. Le corpus issu de cette enquête s'avère par conséquent limité à une dizaine de traces aux caractères fort hétéroclites.

Si la révélation exceptionnelle du jardin à la française de Saint-Symphorien (Sarthe) fait figure d'exception, en revanche, il n'est pas rare que les tracés des avenues donnant accès au château ou des allées ouvrant des perspectives soient reconnus. La persistance des grandes lignes de cette architecture paysagère qui participait à la mise en scène des grandes demeures seigneuriales modernes offre l'occasion de comprendre leurs aménagements successifs, intégrant parfois des héritages médiévaux. 
Bouessay (Mayenne), château des Chenêts

Le contraste est saisissant entre la splendeur passée du château des Chenêts, perceptible sur le plan cadastral de 1828, et l'indigence des vestiges identifiables aujourd'hui. Des différents corps de bâtiments (détruits dès le début du xix ${ }^{e}$ siècle), des avenues d'accès ou des allées ménageant des perspectives, mais aussi bien sûr du gigantesque jardin rectangulaire traversé d'allées perpendiculaires ou obliques, dessiné au nord de la demeure, il ne reste plus aujourd'hui que la base circulaire d'un pigeonnier. Pour l'heure, la photographie aérienne a seulement permis de retrouver les traces de fosses de plantation d'arbres disposés en hémicycle, sur le côté oriental de l'ancien château. Ce plan désigne probablement une structure en demi-lune qui amorçait l'ouverture d'une nouvelle perspective vers le levant. La totalité du parc a été intégrée dans le domaine agricole.



Extrait du plan cadastral de 1828 (tableau d'assemblage).
ณึ

$m$
$\stackrel{m}{N}$
$\stackrel{\vec{N}}{\vec{r}}$

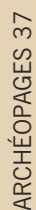
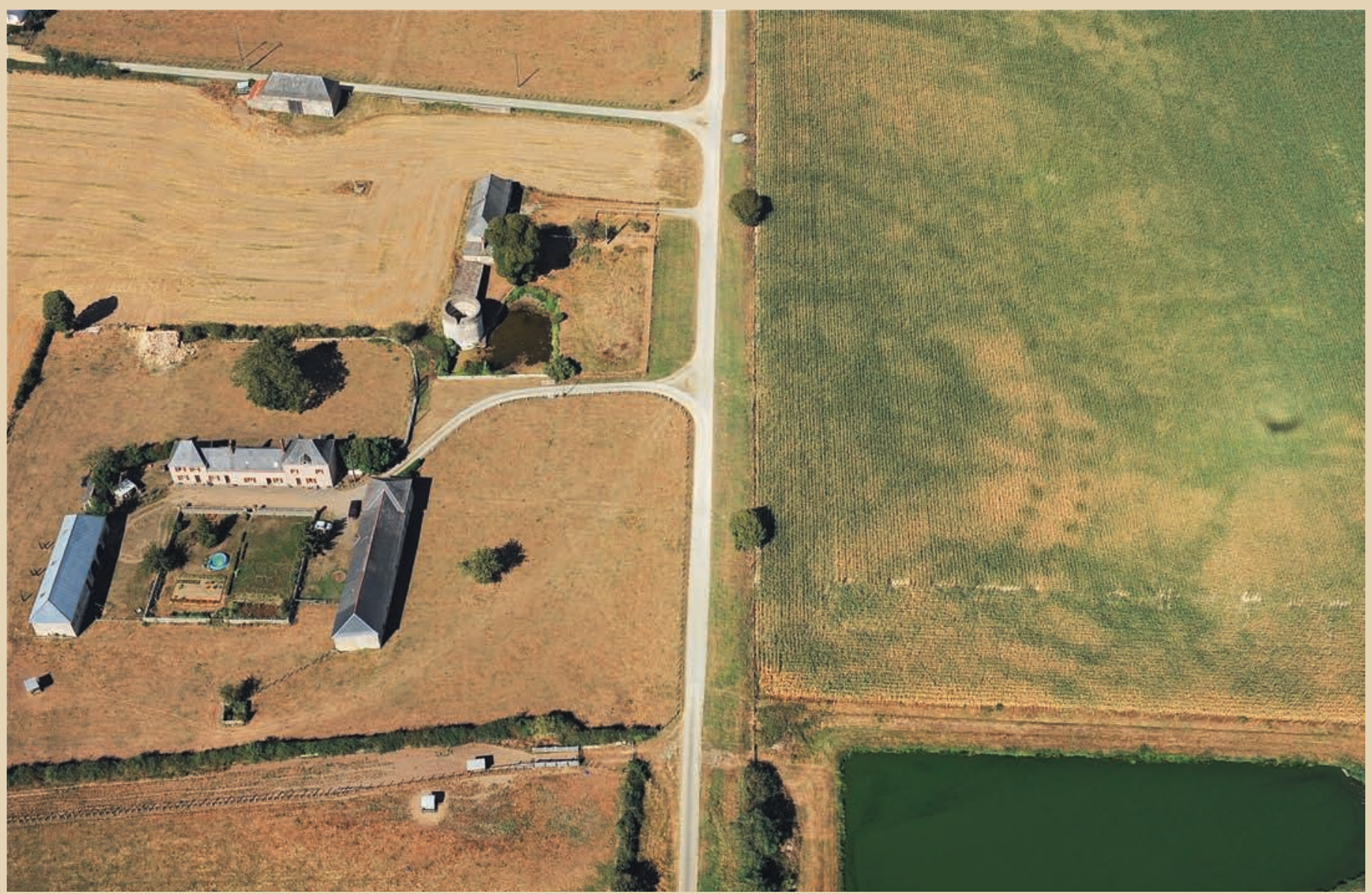

Photographie aérienne du 7 septembre 2012. 


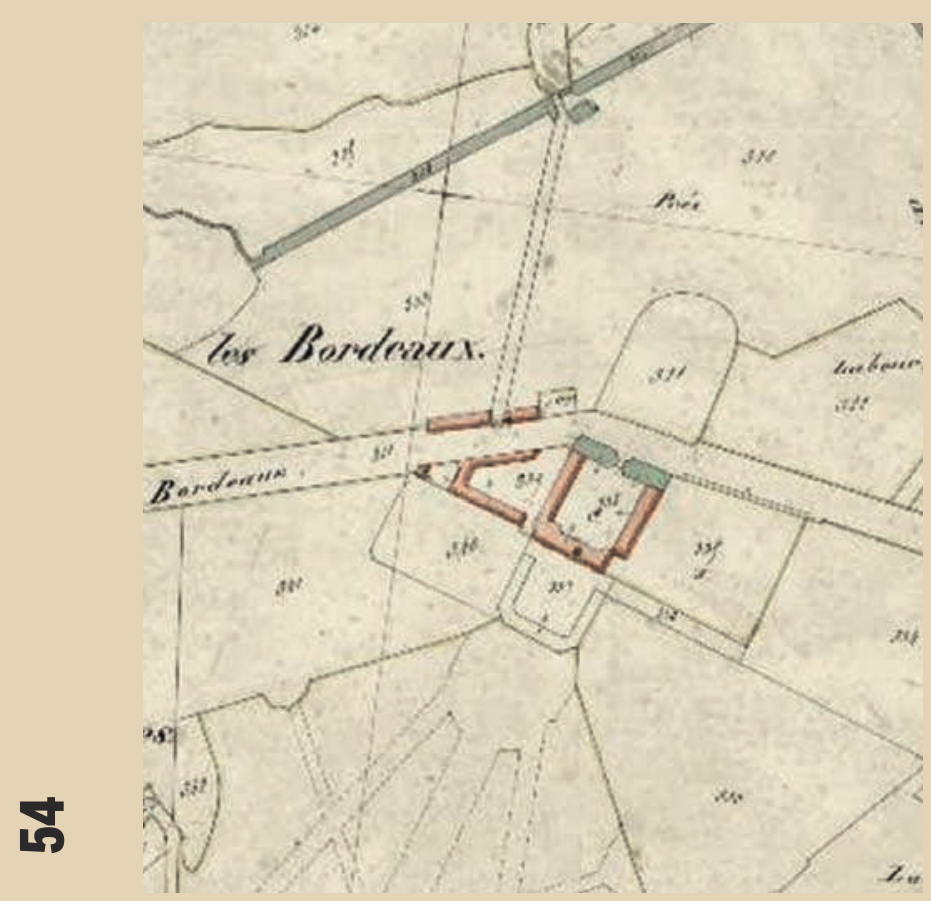

Extrait du plan cadastral de 1833 (section C2).

Amné (Sarthe), château des Bordeaux L'actuel château, construit au milieu du xvirr ${ }^{e}$ siècle, est une demeure d'architecture classique composée d'un corps de logis simple terminé par deux pavillons saillants (Seydoux, 1988, p. 19). Lorsque le plan cadastral est levé en 1833, le château des Bordeaux conserve encore toutes les caractéristiques d'un grand jardin à la française. Deux grandes avenues le reliaient, vers le nord, au village d'Amné et vers le sud, à la route Laval-Le Mans. Le parc, qui se développait essentiellement sur le côté occidental de la demeure (ici, en bas du détail du plan et en haut de la photographie du 7 septembre 2012 ), s'organisait de part et d'autre d'une longue allée principale ponctuée de rotondes aiguillant des allées secondaires entrecroisées. Du côté oriental, une autre allée franchissait le ruisseau de la Doucelle et se terminait par une structure en demi-lune. Enfin, un long canal avait été obtenu. en rectifiant le lit d'un petit ruisseau. Aujourd'hui, si les avenues d'accès au château ont conservé leur usage, la majeure partie du parc a disparu, notamment vers le couchant où elle est en passe d'être occultée par une reprise forestière. L'allée orientale a été détruite lors de l'intégration au domaine agsicole et n'est plus visible qu'exceptionnellement d'avion en période de sécheresse (photographie du 23 juin 2006 ). Quant aux espaces réservés aux jardins, s'ils restent perceptibles au contact du château, ils ont cédé la place à une simple pelouse.

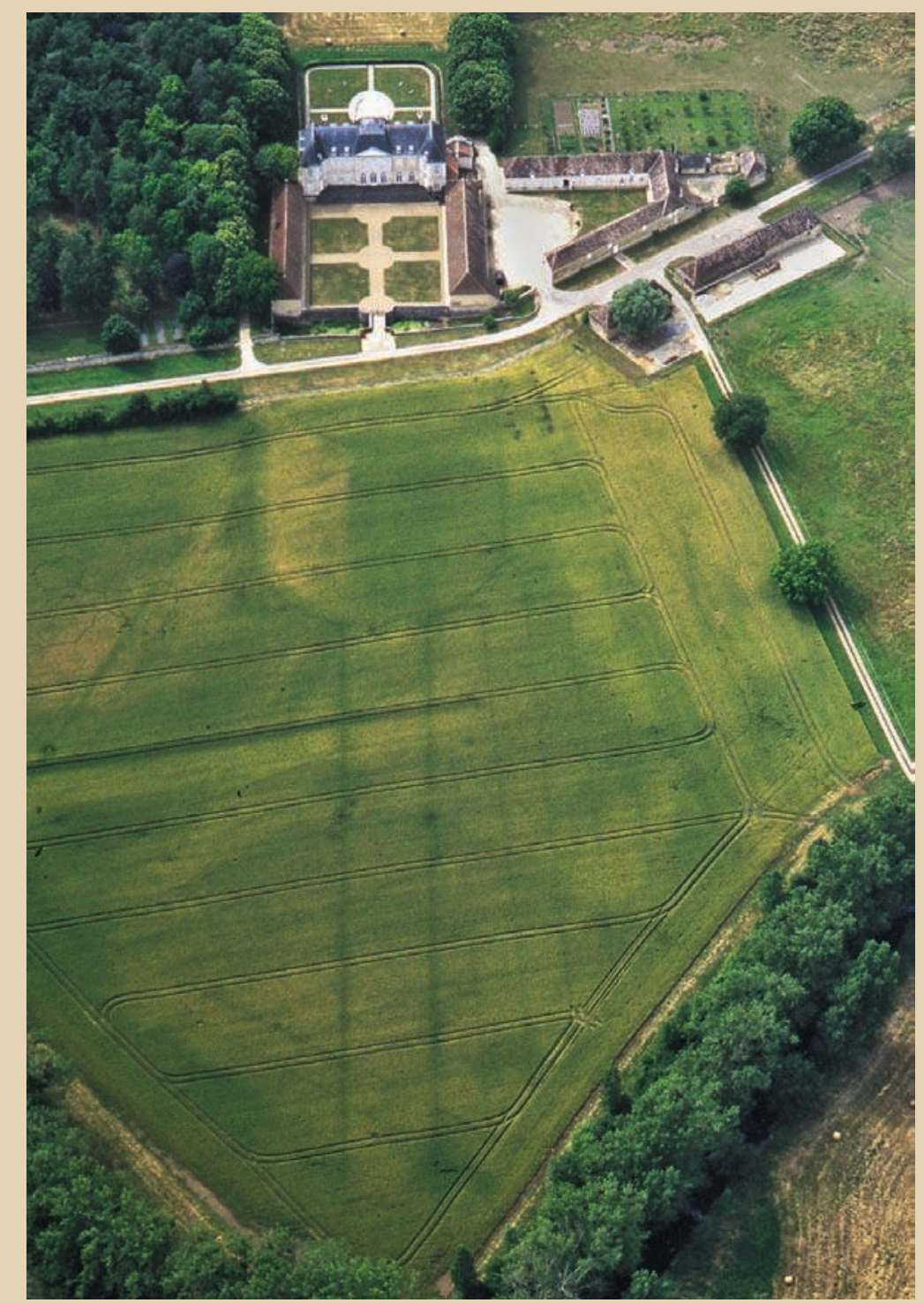

Photographie aérienne du 23 juin 2006.

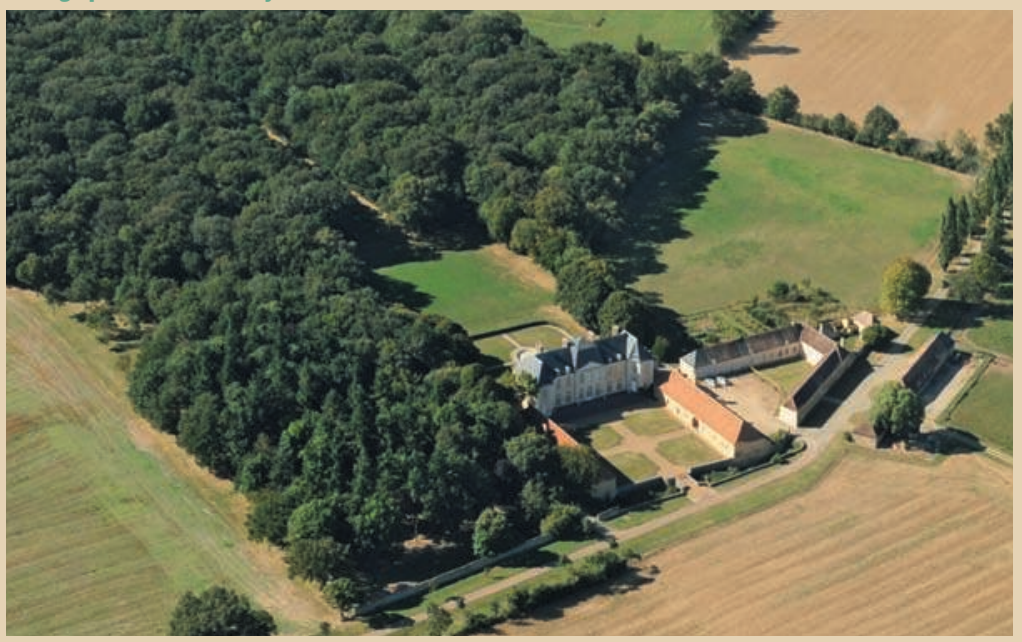

Photographie aérienne du 7 septembre 2012 


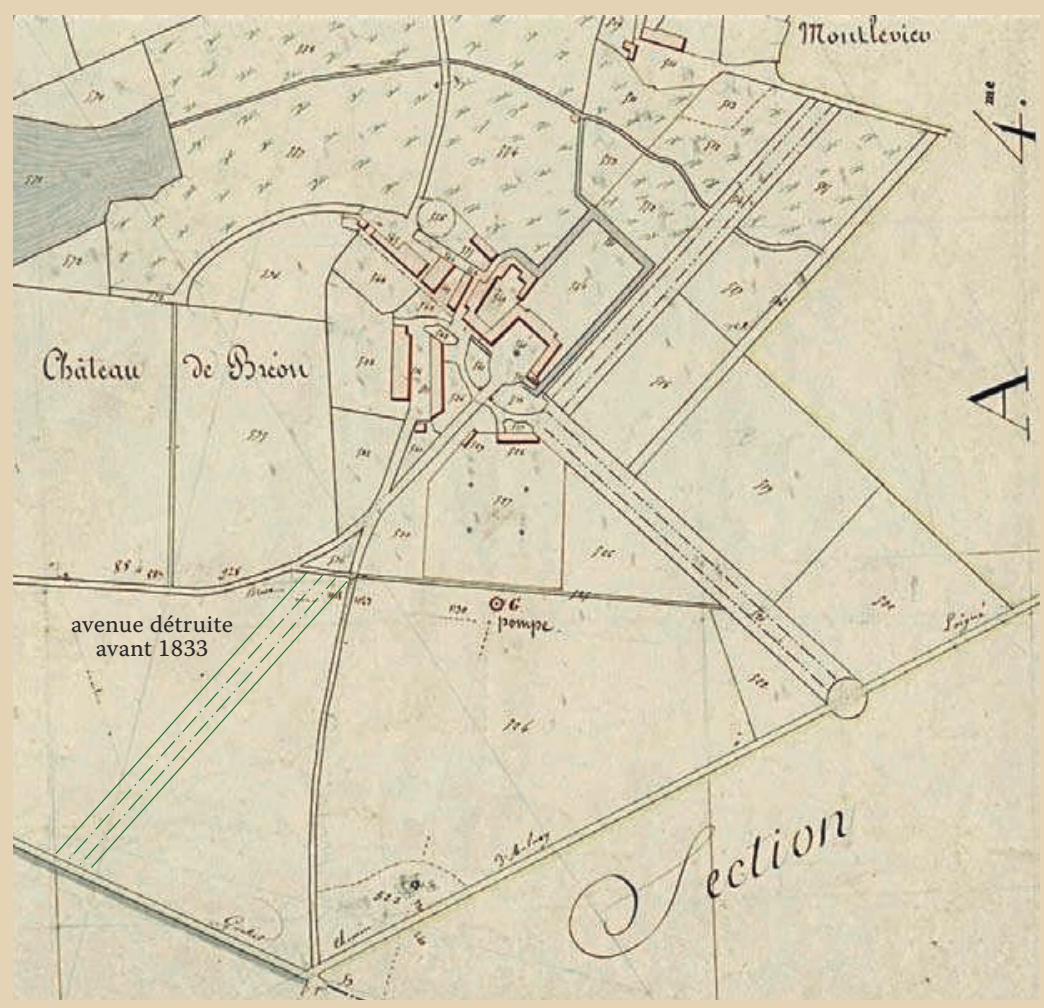

Marigné-Peuton (Mayenne), château de Bréon

Fn 1833, le château de Bréon possède encore de nombreux attributs d'un grand parc à la française, organisé en fonction de deux axes perpendiculaires dont le château constitue le cœur. Deux larges avenues, tout en permettant l'accès à la demeure, ouvrent des perspectives vers le nord-est et le sud-est. Le barrage du ruisseau de Bréon permet

l'alimentation de bassins dont le plan est calqué sur celui du corps de logis. Le quadrilatère formé par ces derniers devait être réservé au jardin. L'adjonction, sans doute tardive, de nombreux bâtiments à vocation agricole ne modifie pas le plan originel de la propriété. L'apport de la photographie aérienne consiste en la reconnaissance de l'avenue principale qui débouchait sur la cour d'honneur du château et se terminait par une structure en demilune encore perceptible sur le cadastre napoléonien. Celle-ci, déjà détruite en 1833 , est matérialisée par une piste de circulation centrale, encadrée par des fossés bordiers intérieurs et de larges fossés extérieurs qui délimitaient son emprise foncière maximale. L'examen aérien des abords des édifices actuels a également permis de retrouver les fondations de plusieurs dépendances du château, ainsi que l'assiette circulaire d'une motte préexistante.

Extrait du plan cadastral de 1833 (section A3).






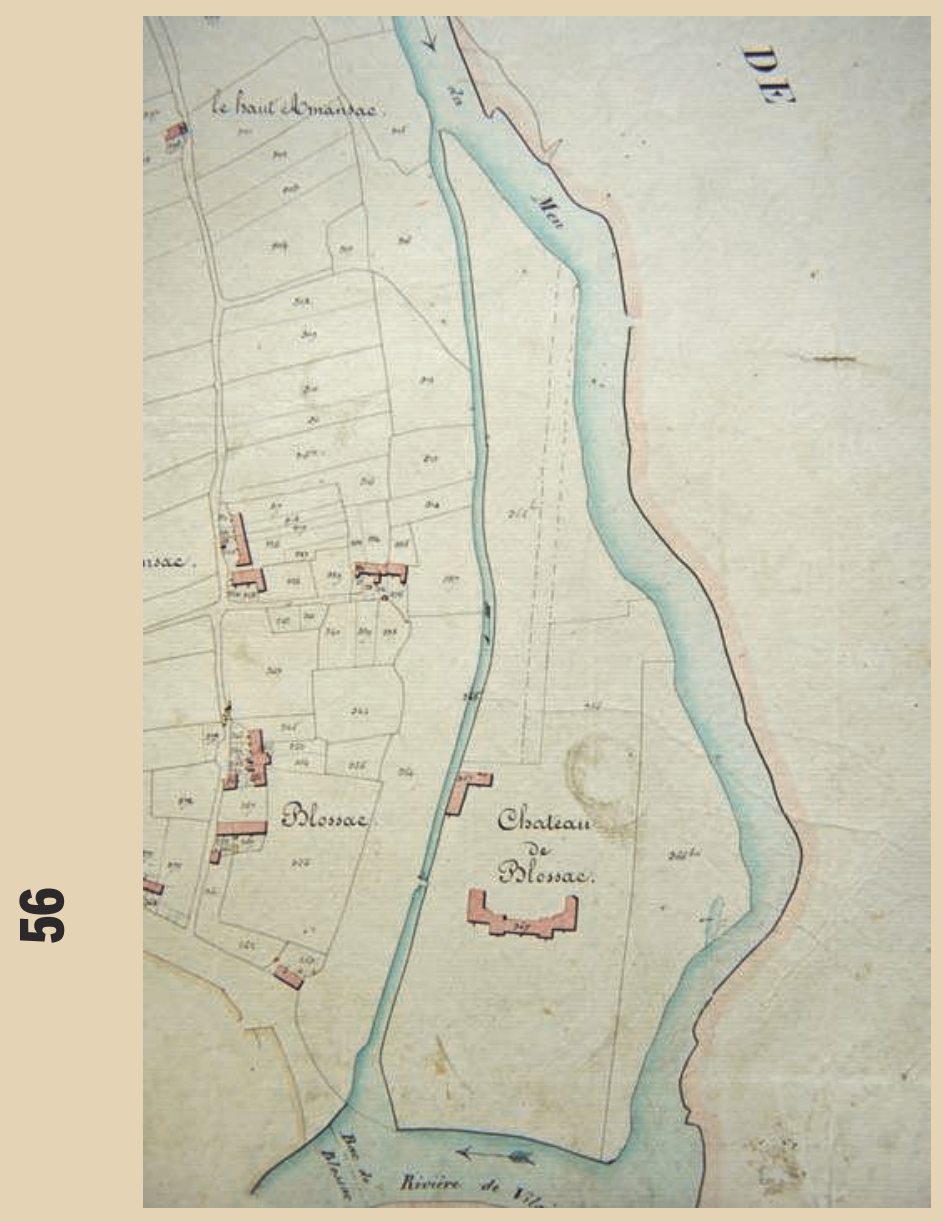

Goven (Ille-et-Vilaine), château de Blossac

Le château moderne de Blossac, achevé en 1678, succède à un manoir du xvi ${ }^{\mathrm{e}}$ siècle. À la confluence de la vilaine et du Meu, en situation insulaire naturelle (Banéat, 1927-1929, II, p. 117-118), la topographie des lieux offre un terrain plat et la présence permanente de l'eau, propices au dessin d'un parc arboré. La forme longiligne de l'île est mise à profit par une avenue d'orientation nord-sud axée sur le château. La photographie aérienne permet de s'assurer que cet axe est repris sur la face méridionale du château par une allée centrale qui détermine le plan symétrique d'un jardin à la française découpé en quatre surfaces rectangulaires égales.

Extrait du plan cadastral de 1832 (section D2).

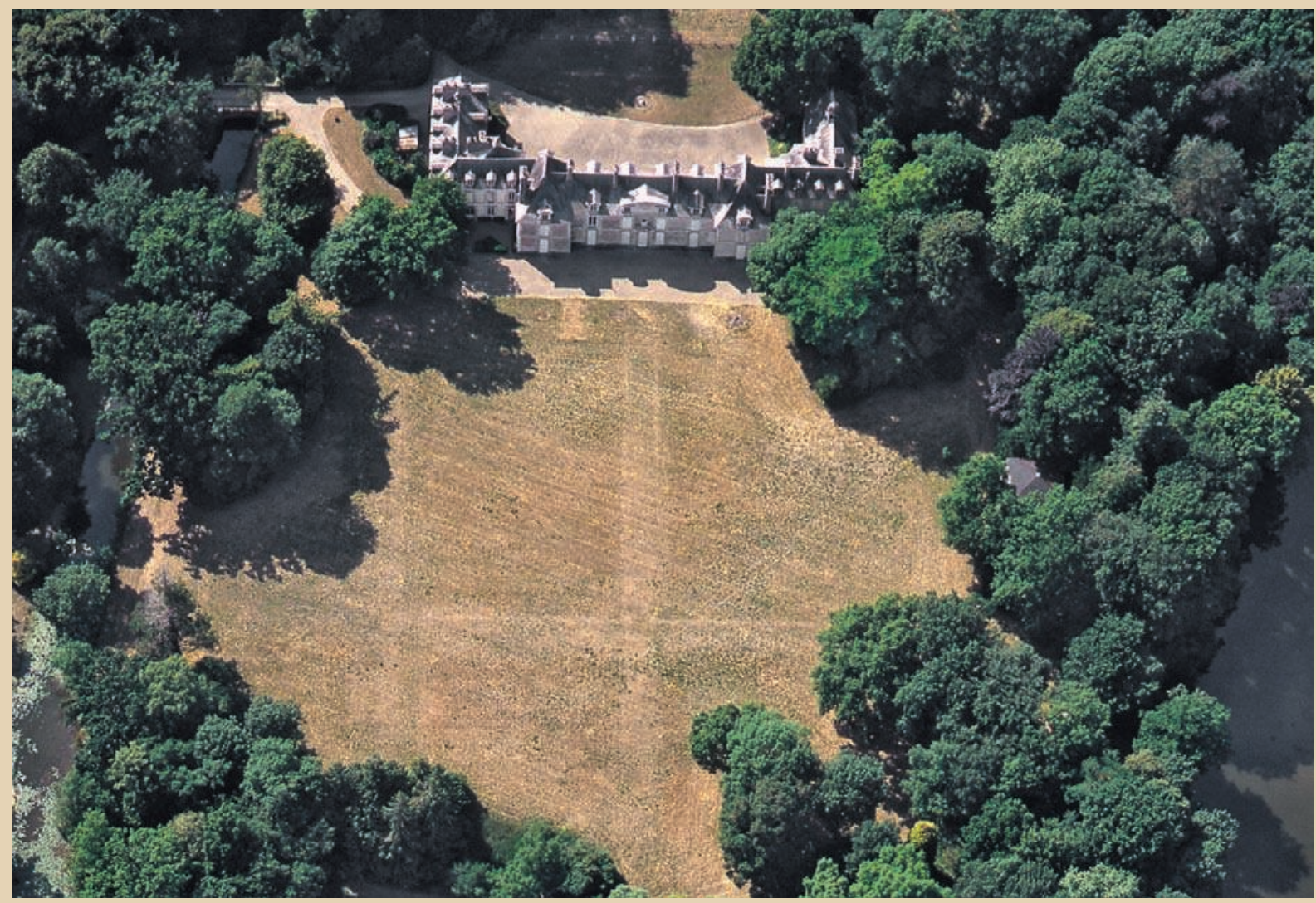

Photographie aérienne du 3 juillet 2001. 
Sainte-Gemmes-d'Andigné (Maine-et-Ioire), château de la Blanchaye

Le plan cadastral de 1826 livre un état du château et de son parc antérieur aux bouleversements de la seconde moitié du xrx ${ }^{e}$ siècle. I'édifice est construit sur un versant qui domine un méandre de la Verzée. Son accès, depuis la route qui mène du bourg de Sainte-Gemmes à celui d'Iré, s'effectuait par une avenue terminée par un parterre en demi-lune. Vers le nord, un jardin potager de plan quadrangulaire était protégé par un mur et accompagné d'un bâtiment rectangulaire correspondant sans doute à une serre. L'environnement paysager actuel du château est à peine reconnaissable : les jardins d'agrément ont laissé place à un simple découpage géométrique de plates-bandes engazonnées et la grande avenue a été abandonnée. Fnfin, le dessin de la structure de clôture du potager et des fondations de sa serre n'est plus identifiable que sur une photographie aérienne du 2 juillet 1996.

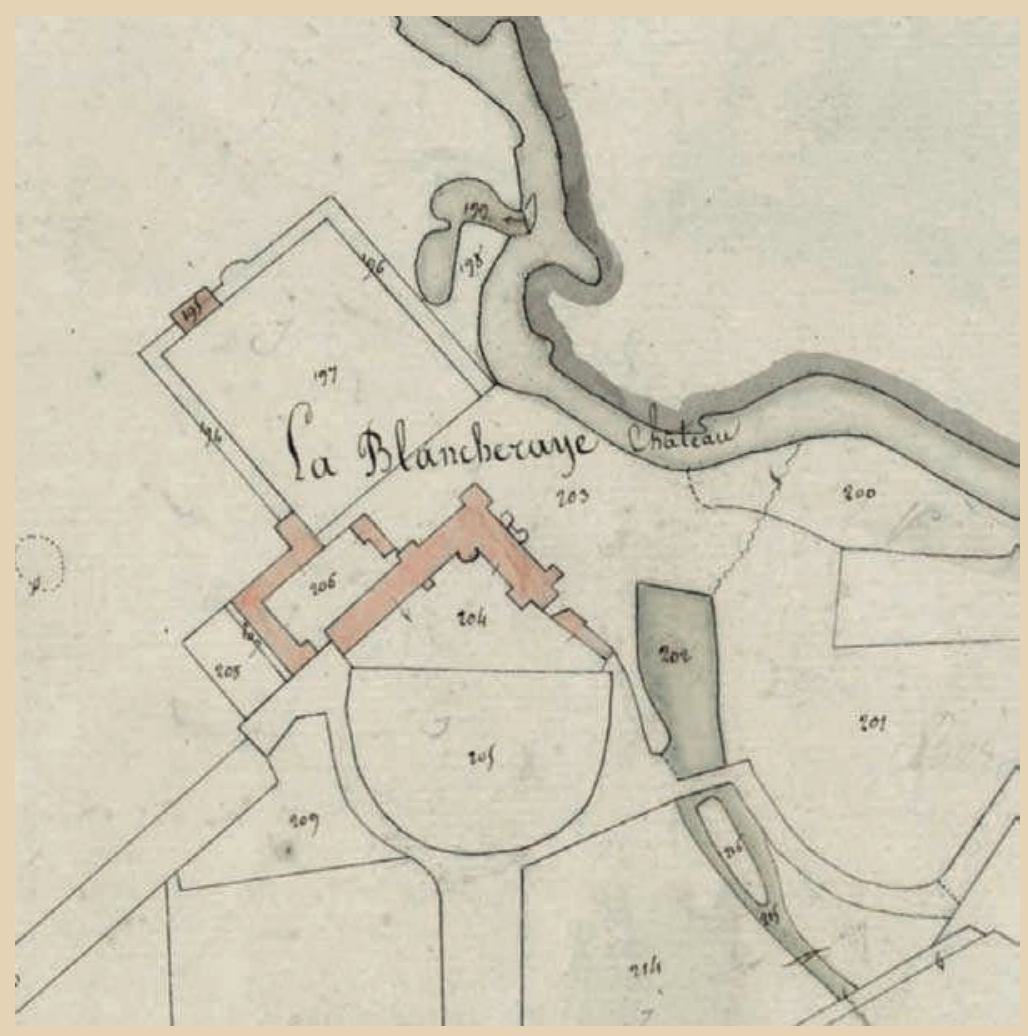

Extrait du plan cadastral de 1826 (section B2).

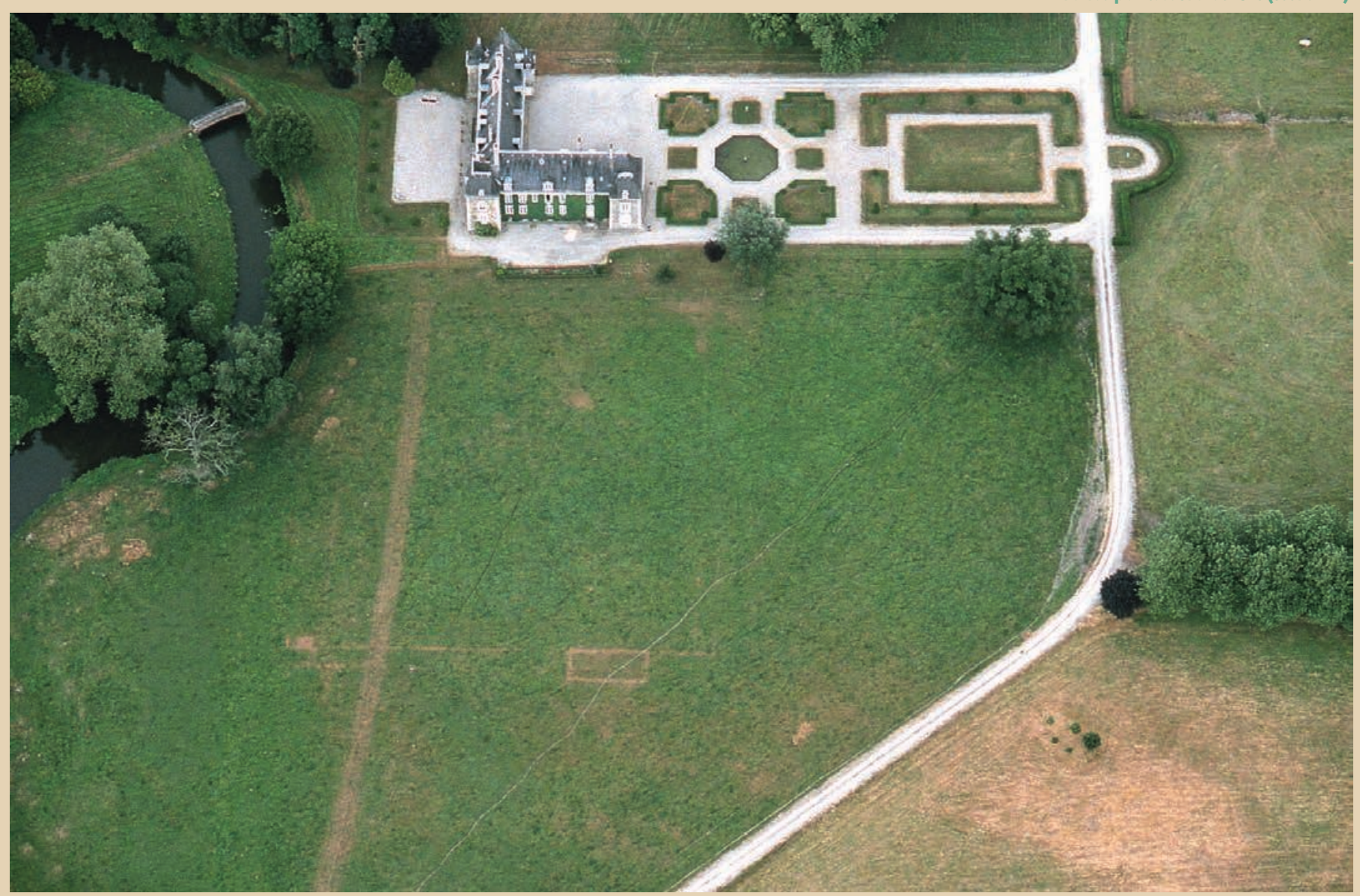




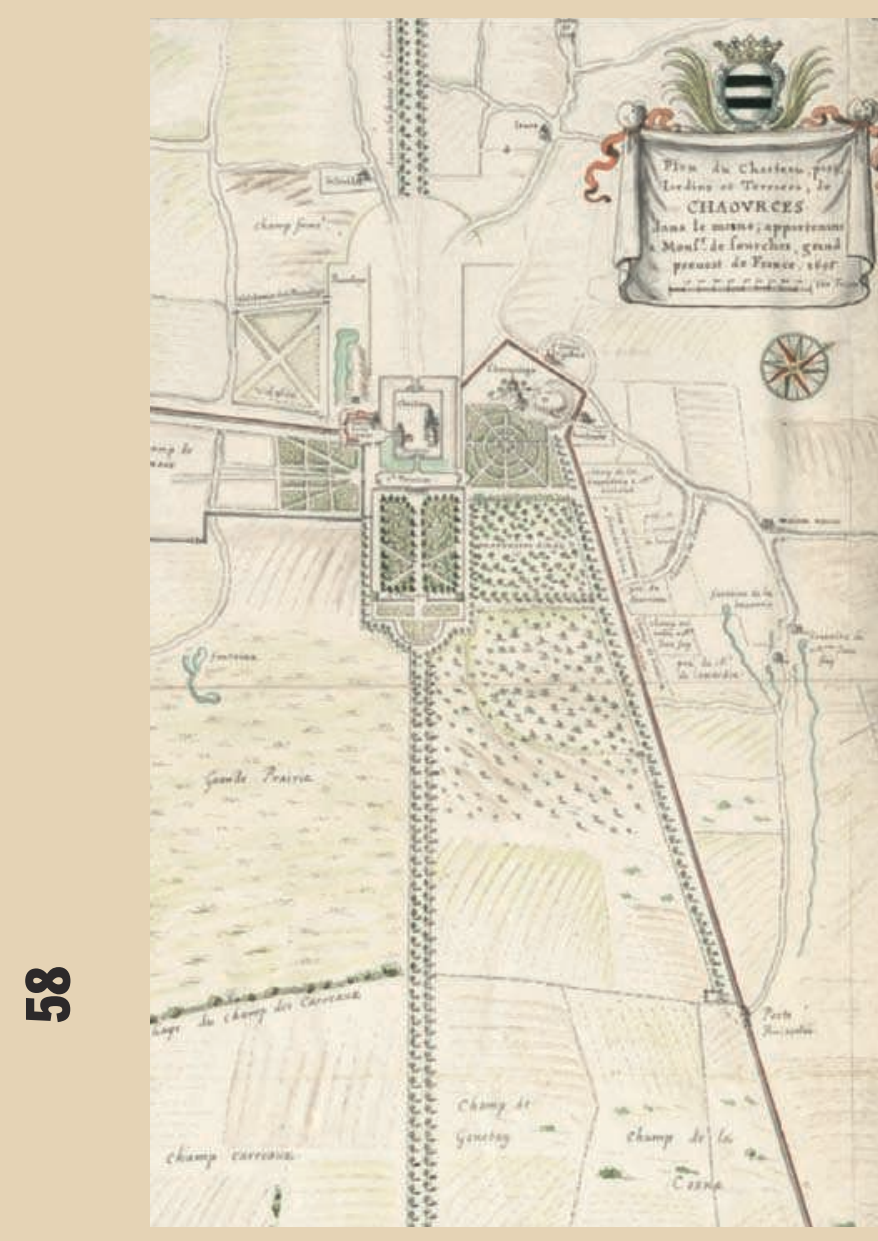

Une vue d'ensemble et un détail de l'aquarelle de Louis Boudan, 1695, « Plan du chasteau, parc, jardins et terraces de Chaources, dans le Maine, appartenant à M. de Sourches, grand prevost de France ».

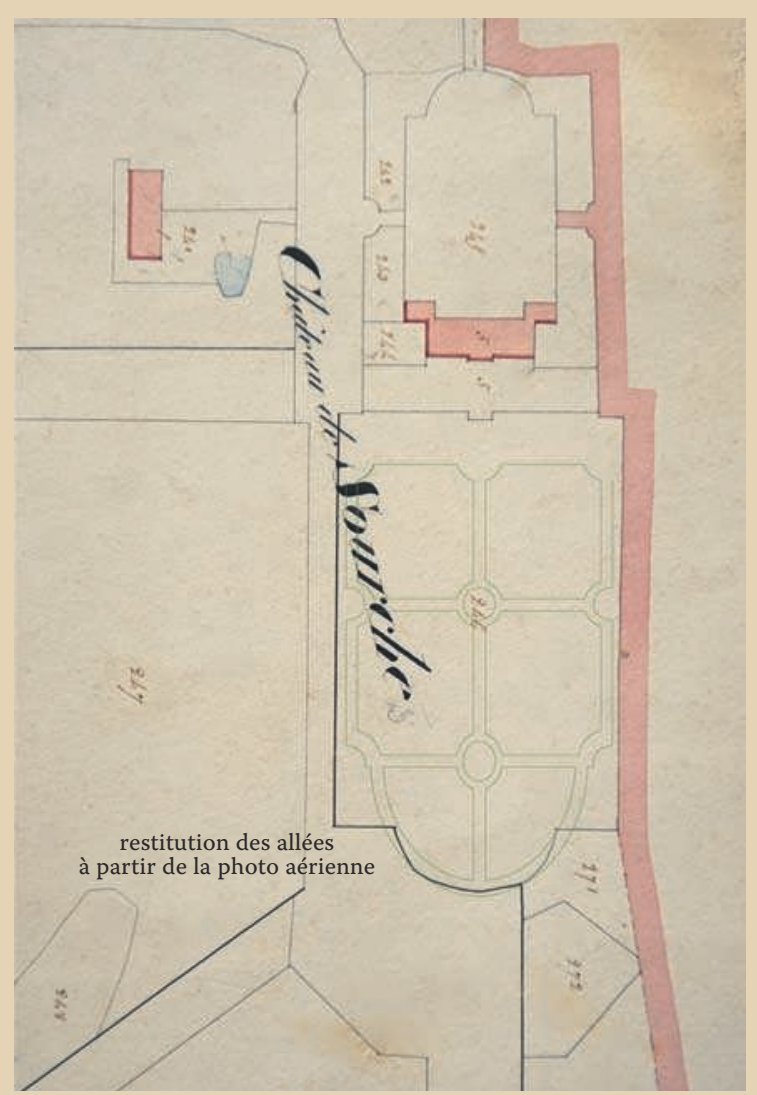

Extrailt du plan cadastral de 1830 (section B2).

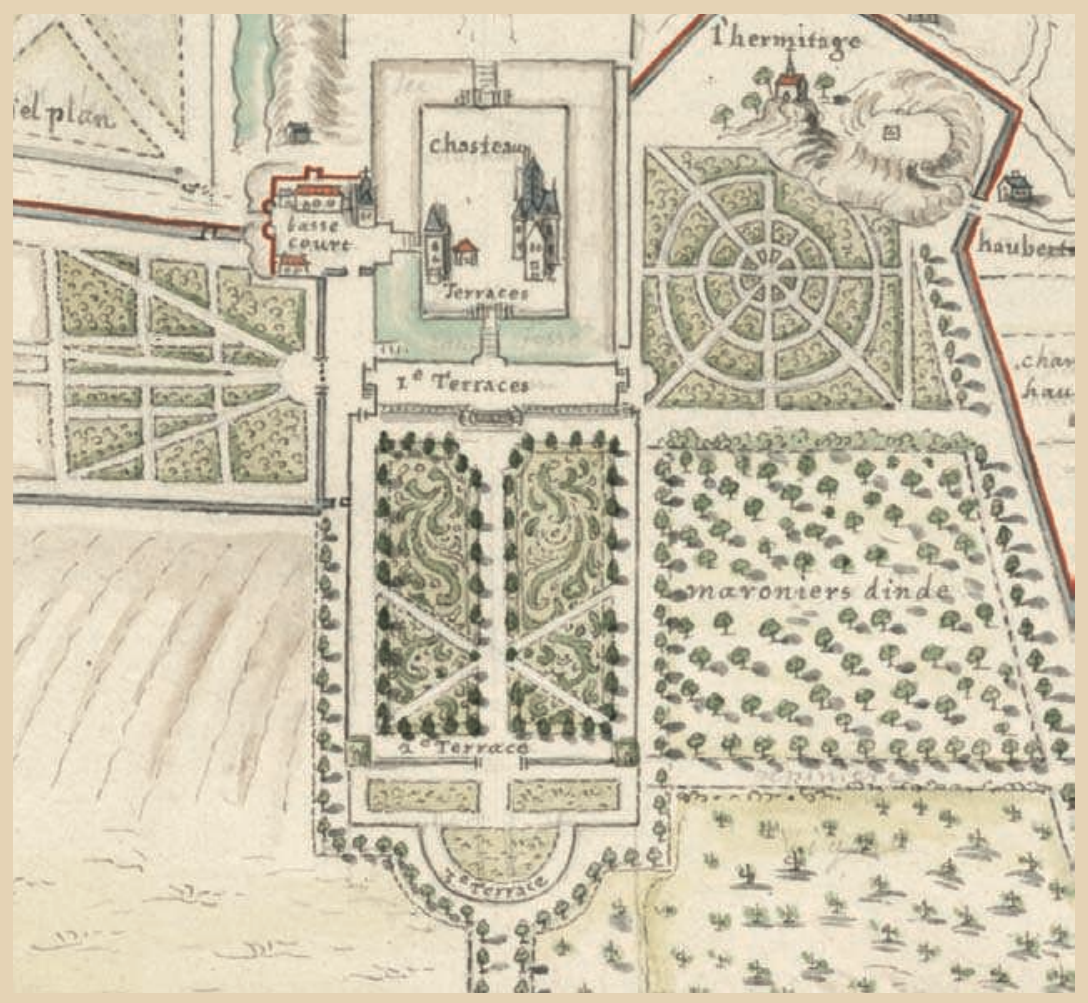

Saint-Symphorien (Sarthe), château de Sourches

L'aménagement du parc du château de Sourches est à la mesure de ses propriétaires successifs, marquis de Sourches et ǵrands prévôts du roi de France pendant les xvir et xvIr' ${ }^{e}$ siècles.

Une aquarelle de Louis Boudan, réalisée en 1695, montre la magnificence de la propriété. Le vieux château, bâti à l'intérieur d'un vaste quadrilatère défini par de larges douves, est encadré, à l'ouest, par une cour d'honneur, à l'est, par une terrasse et un jardin à la française, au nord, un labyrinthe, un potager, des vergers, et enfin, les reliefs de l'ancienne motte et de sa basse-cour intégrés à l'aménagement général. Le jardin oriental, rythmé par trois terrasses, s'inscrit dans un rectangle traversé par une allée centrale qui distribue symétriquement les parterres où s'épanouissent les dentelles de topiaires. Dès cette époque, des avenues perpendiculaires longues de plusieurs kilomètres s'étendent autour du château. Si la construction du nouveau château dans un style néoclassique, entre 1761 et 1786, par l'architecte du roi Gabriel de Lestrade, pour Louis II du Bouchet, ne bouleverse pas l'ordonnancement du parc dessiné par Jules Hardouin Mansart, il semble néanmoins acquis que son jardin à la française ait été totalement remodelé. C'est, du moins, ce qu'atteste notre photographie du 28 juin 2004 sur laquelle le plan complet et détaillé d'un jardin différent de celui de l'aquarelle de 1695 est réapparu à la faveur d'un regain d'herbe après fenaison. Son plan reste géométrique et adopte une symétrie parfaite de part et d'autre d'une allée centrale qui distribue les allées secondaires et les parterres. Les activités agricoles actuelles continuent d'évoluer dans le cadre du xvir ${ }^{\mathrm{e}}$ siècle. 

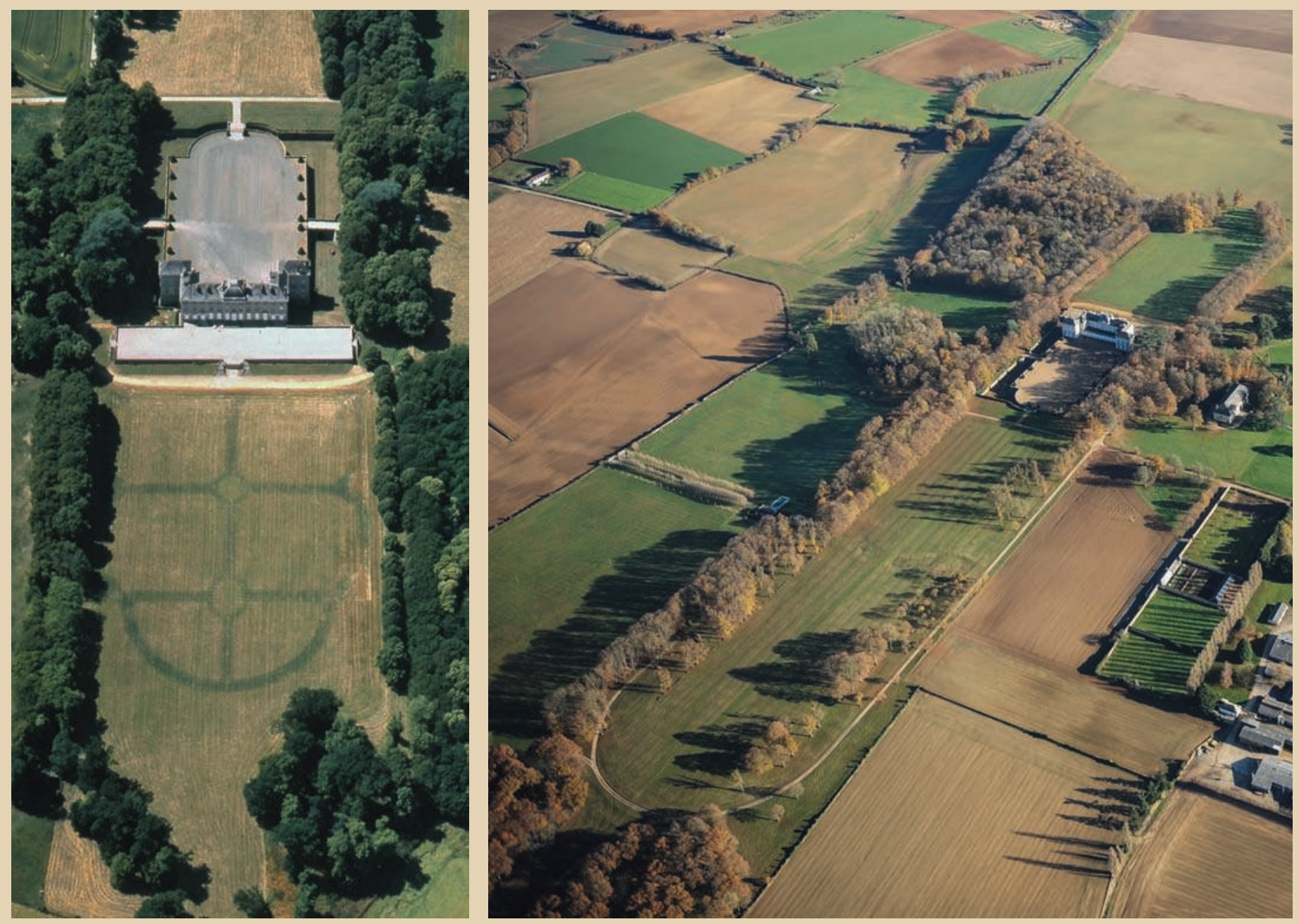

Photographie aérienne du jardin à la franç̧aise, du 28 juin 2004.

Photographie aérienne d'ensemble du 03 décembre 2013.





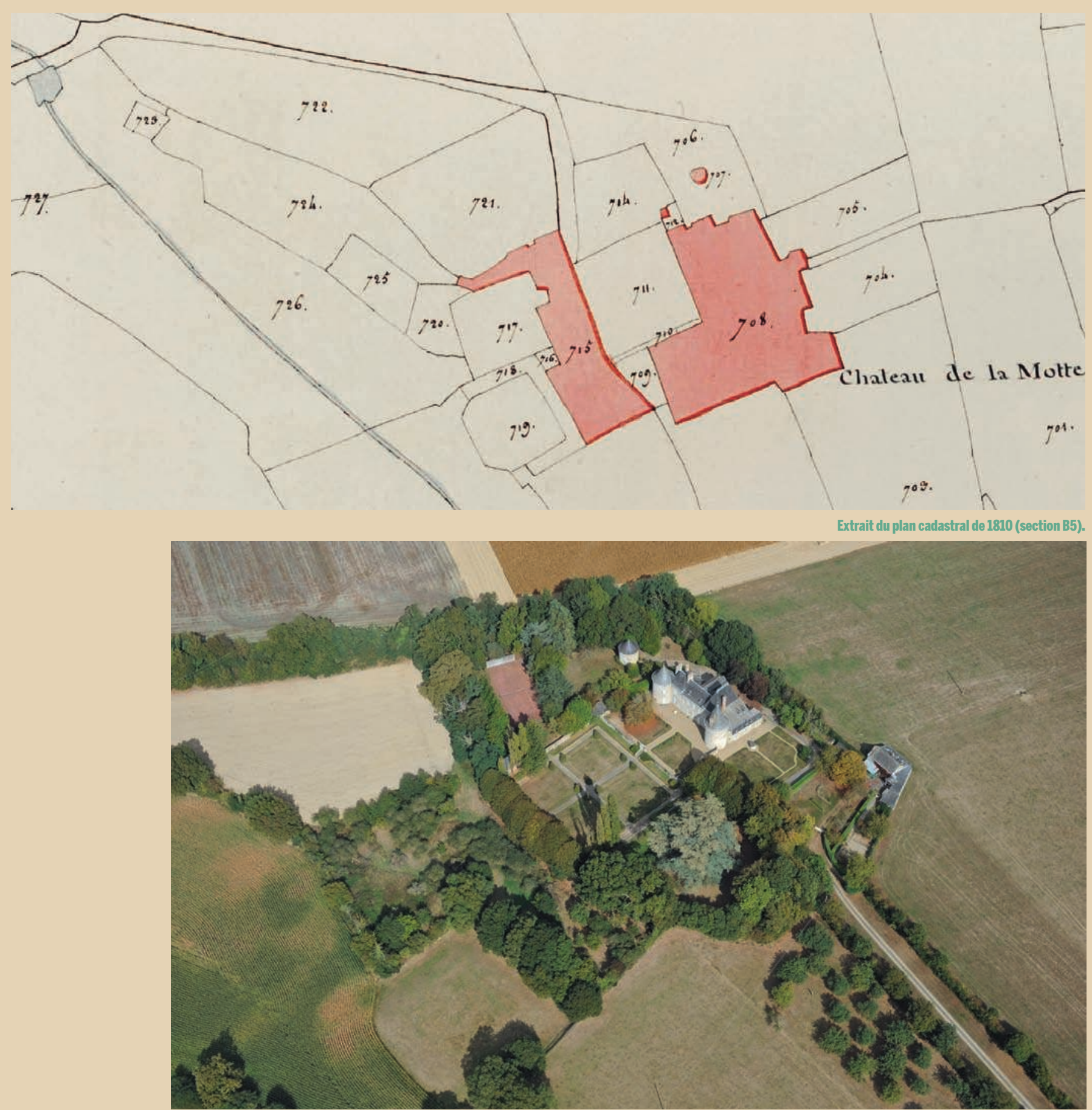

Photographie aérienne du 2 octobre 2009.

Gennes-sur-Seiche (Ille-et-Vilaine), château de la INotte

Le château de la Motte, construit au xvi ${ }^{\text {e }}$ siècle et restauré à la fin du xvII' siècle, se compose d'un corps de logis flanqué d'un pavillon carré et de deux grosses tours rondes, tandis que son pigeonnier est excentré vers le nord (Banéat, 1927-1929, II, p. 91). L'empreinte des douves, en partie comblées, se retrouve dans un plan trapézoïdal délimité par des arbres, au sein duquel s'insèrent le bâtiment et son parc moderne. Cet ordonnancement n'a pas totalement occulté un plan plus ancien caractérisé par un découpage régulier de plateformes, dont la disposition et les superficies semblent reproduire le plan quadrangulaire d'une motte installée en contrebas vers l'ouest, au contact d'un ruisseau. Ici, point de grandes perspectives, tout le décor évolue de manière ramassée. Le château, planté sur la ligne haute d'un versant, domine son domaine jusqu'à la motte (ici, parcelle ' 719 sur le plan et en bas de la photo), installée au fond d'une petite vallée, qui fait partie intégrante de l'ensemble paysager. Cet ensemble qui a passé sans encombre l'épreuve du remembrement des années 1970 , constitue un exemple insoupçonné d'un paysage modelé au xvi ${ }^{\mathrm{e}}$ siècle. 


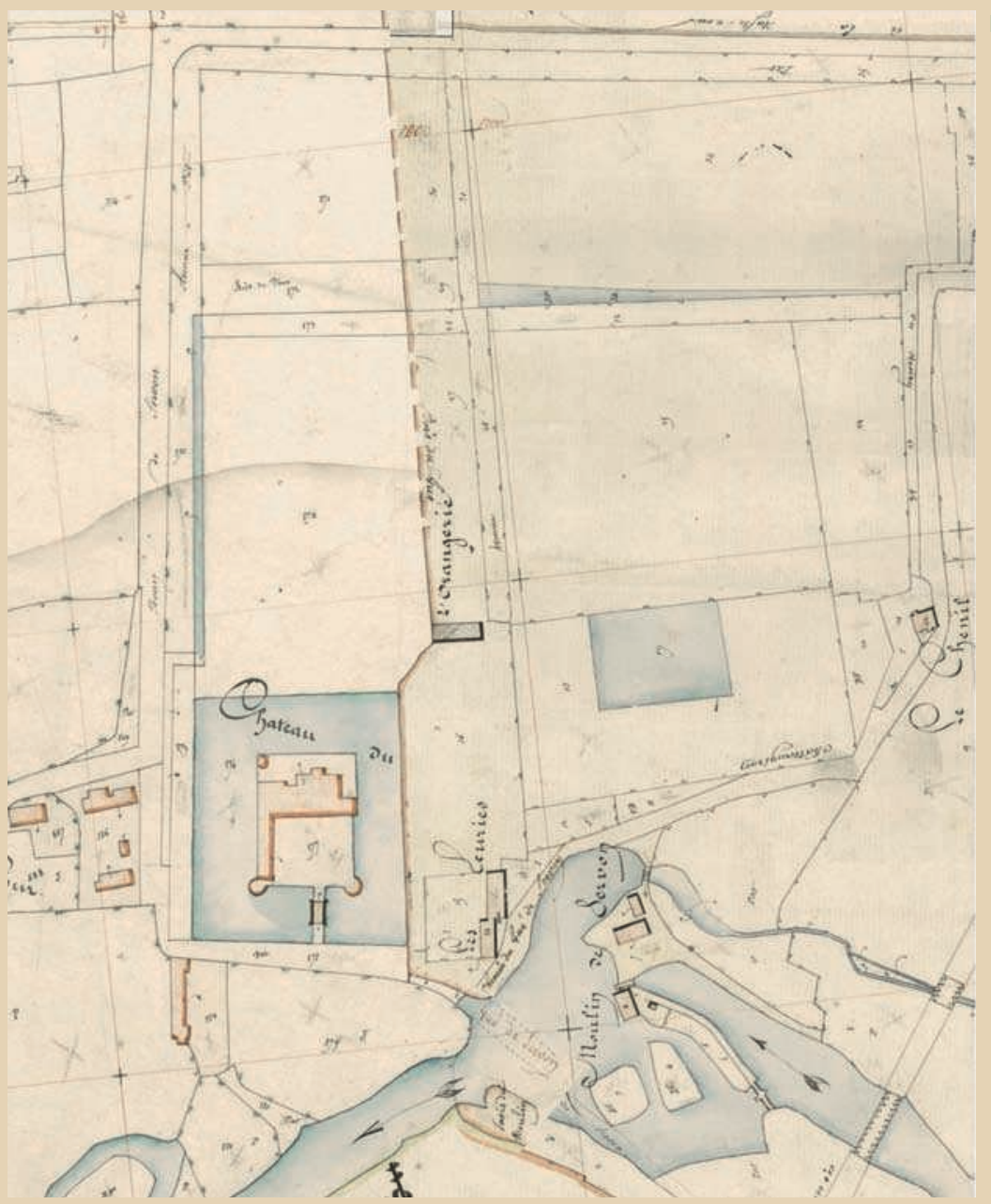

Extrait du plan cadastral de 1849.

Servon-sur-Vilaine (Ille-et-Vilaine), château du Gué

L'installation d'une fortification à proximité immédiate de la vilaine trouve son origine dans la volonté de contrôler un passage à gué du fleuve. L'ancien manoir du xv' siècle, restauré au xvIr siècle, était flanqué de quatre tours rondes (Banéat, 1927-1929, IV, p. 187-188). Une douve rectangulaire démesurée protège le château tout en le mettant en scène. La photographie aérienne a permis de retrouver dans les moindres détails le plan des structures enregistrées sur le cadastre de 1849 au nord-est de la demeure. Une série d'allées dessinent un vaste quadrilatère et permettent l'accès à plusieurs bâtiments rectangulaires qui correspondent aux écuries et à l'orangerie.

Un vivier maçonné, de plan rectangulaire, s'inscrit dans cet ensemble.

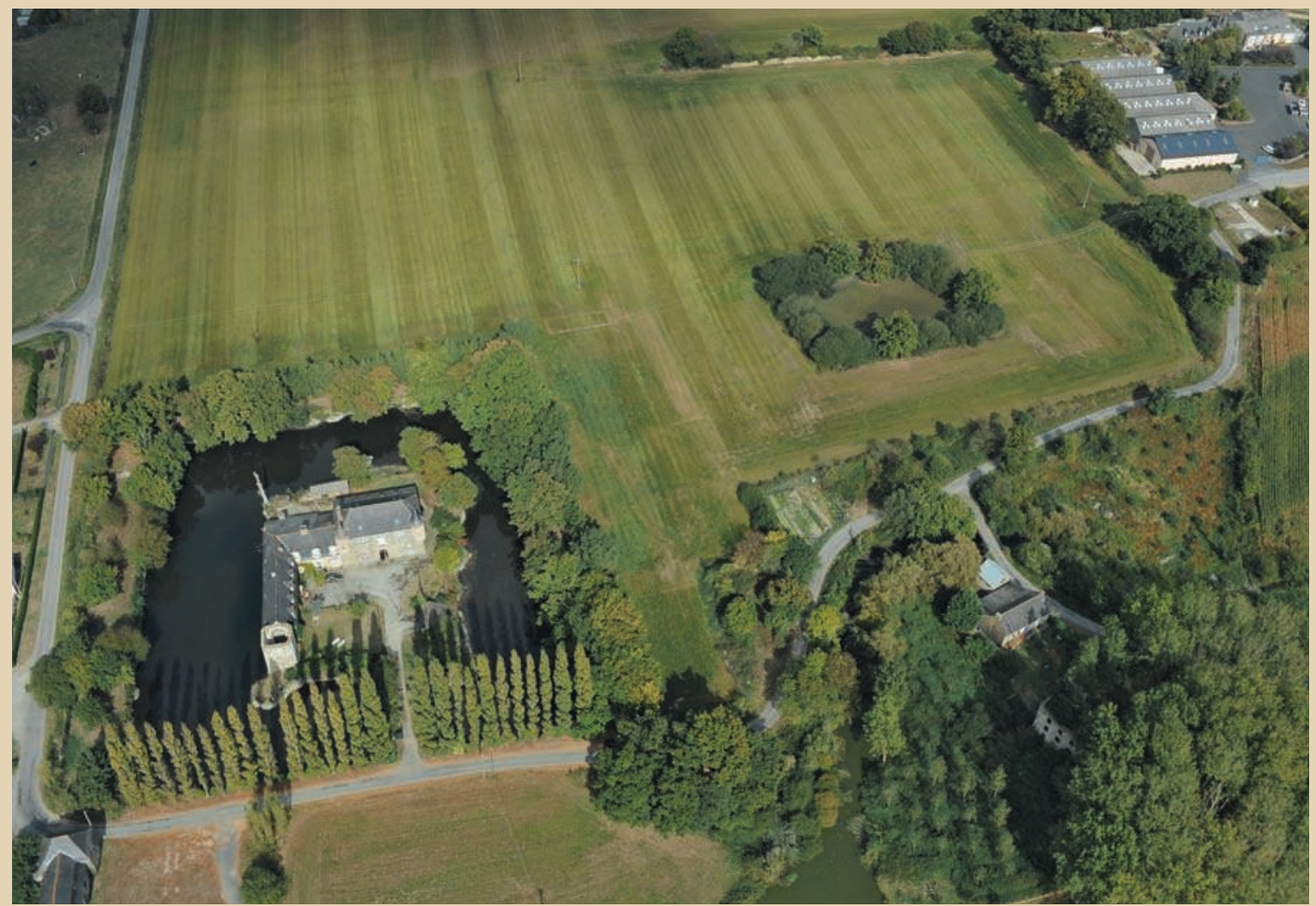

